\title{
Conductivity Percolation on a Square Lattice with Core-Shell Particles
}

\author{
Kazuhito Shida, Ryoji Sahara, MN Tripathi, Hiroshi Mizuseki and Yoshiyuki Kawazoe \\ Institute for Materials Research, Tohoku University, Sendai 980-8577, Japan \\ Particles made by coating an insulating core with a thin layer (shell) of conducting materials, called core-shell particles, are recently \\ proposed to realize materials with particular nano-scale structures. Such structures work as a "porous media" of electric current, and its modified \\ characteristics may be useful to improve some materials, for example the transparent conducting films. \\ In this report, the basic feasibility of this idea is tested in the framework of the percolation model and the random register network model. \\ The observed critical behavior and critical exponents of conductivity are also discussed, for the first time for $2 \mathrm{D}$ situations with core-shell \\ particles. [doi:10.2320/matertrans.M2009329]
}

(Received October 9, 2009; Accepted January 21, 2010; Published March 3, 2010)

Keywords: thin film conductor, percolation model, critical phenomenon, granular material, core-shell particles

\section{Introduction}

The percolation model is a simple and powerful theoretical tool which plays an important role in explaining various physical phenomena related to properties of disordered systems. In this report we mainly consider the site-percolation model, in which the characteristics of the sites exhibit a quenched randomness: usually, sites are randomly occupied at probability $p$. At a critical threshold $p_{\mathrm{c}}$ of the site occupation probability, a phase transition regarding interconnectivity occurs from an unpercolating phase to a percolating phase, where at least one passages through occupied sites are available between almost all pairs of occupied sites. The behavior of the criticality shows a strong "universality" (independence from the type of the lattice except for the dimensionality). The strength of such percolation models comes from their abstractive nature: the detailed characteristics of the passage like width and length is completely omitted from the model.

However, these characteristics should be reintroduced to the model when percolation models are considered on a realistic material. That is, the electric conductance of percolation cluster is dependent not only on the existence of the large cluster, but also on the "width" and "length" of the passage of the electric current defined in the percolating cluster. Introducing of such a physical detail is useful in analyses of some applications where conducting materials must simultaneously satisfy special characteristics other than the electronic conductance, because such materials tend to be less abundant. A good example of such a special characteristics is the optical transparency; Transparent conducting films are materials of great technological importance because they are necessary in making flat panel displays or solar cells. Regarding sustainable production of such films with required level of conductance, deposition methods based on nanoscale particles ${ }^{1-5)}$ have been a focus of intense interest. Such a particle based film production is strongly relevant to the 2D percolation model investigated in this report, where a systematic optimization of the "width" is considered.

In a previous work, ${ }^{6,7)}$ we proposed and examined a new type of $2 \mathrm{D}$ site-percolation model where two different sizes of particles, i.e., particles $\mathrm{A}$ and $\mathrm{B}$, are present on a square lattice. In the model, the smaller particle (particle A) can occupy one site of the lattice just as in the ordinary model, whereas the larger particle (particle B) can occupy multiple sites. Lattice sites are occupied, either by particle A or by a part of particle $\mathrm{B}$, with overall probability $p$, that is the sum of site occupation probabilities of particles $\mathrm{A}$ and $\mathrm{B}$, denoted respectively as $p_{\mathrm{A}}$ and $p_{\mathrm{B}}$ :

$$
p=p_{\mathrm{A}}+p_{\mathrm{B}} .
$$

Despite the clear difference from the standard model where the particle size is uniform, in our previous work where $p_{\mathrm{A}}=p_{\mathrm{B}}$ is assumed (this model is called "binary distribution model" in this paper), the conductance of the system did not exhibit a large deviation from that of the standard percolation model. When $p_{\mathrm{A}}$ is not equal to $p_{\mathrm{B}}$, stronger dependence of $p_{\mathrm{c}}$ on the particle size distribution is reported to be possible, ${ }^{8,9)}$ and the conductance may be affected as well.

In this paper, we further extended the standard percolation model to deal with the particles with core-shell structure. The core-shell particles are comprised of the surface made of a highly conducting (and perhaps expensive) materials and the core made of a less conducting (and perhaps more affordable) materials. The core material can be completely insulating, if the material suffices other important characteristics like transparency. The core-shell structure is attracting strong interest ${ }^{10-12)}$ because it is a simple and promising approach to design a material with required characteristics (in this case, conductivity) while saving resources. However, the absolute level of conductance must be carefully discussed because the conducting "clusters" affected by the shell might not have enough "width". The electric conductance of such system is interesting also in theoretical respect because it may have a different exponent from normal percolation transition. The following is, to the authors' best knowledge, the first report on the electric conductance on a model of 2D granular materials including core shell particles.

\section{Method}

The electric conductance of percolating cluster $^{13)}$ formed on a $2 \mathrm{D}$ square lattice was calculated considering a $2 \mathrm{D}$ 
A

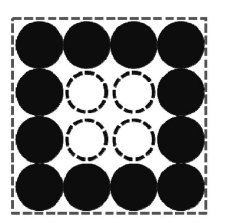

B

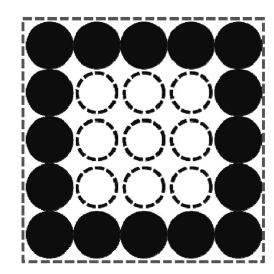

\section{: Conductor sites : Insulator sites}

Fig. 1 In this work, these core-shell type particle designs are used to simulate the core-shell particles with two different sizes $(2 \times 2$ and $3 \times 3)$ of the core. The filled circles are individual conducting sites and the open circles are individual insulator sites that constitute the core.

random resistor network on a square lattice where the value of resistance is $K$ between two conducting sites, and 0 elsewhere. All lattice sites are either conducting or insulating. The conducting sites are placed at either of the surface of randomly placed core-shell particles introduced in Fig. 1 or randomly placed particles with normal sizes. The insulating sites are placed at anywhere conducting sites are not placed (see Fig. 2). As in "binary distribution model", the density of all conducting sites is denoted as $p$. However, in the coreshell model, it is convenient to define the volume fraction of all particles with any type of conductance, $q$. Note that the volumes of the insulating cores are included to $q$, but insulating sites outside the shells are not (instead, they are regarded as normal size insulator particles). For example, by comparing the result of a core-shell model $(q=0.5)$ and binary model $(p=0.5)$, we can quantify the effect of the insulting core in a direct manner. To enable such a comparison, all core-shell calculations were carried out in a condition that the normal particles and core-shell particles occupy the same number of lattice sites (corresponding to $p_{\mathrm{A}}=p_{\mathrm{B}}$ in "binary distribution model"). In Fig. 2, a typical particle configuration generated under $q=0.65, p=0.5$ is illustrated.

The largest connecting cluster of conducting sites that "bridge" the left and right edges of the lattice is calculated by a standard cluster algorithm. Then the Kirchhoff equations are solved on the resister network formed by the largest cluster of conducting sites by means of a simple relaxation method to calculate the total current flowing into the left edge of the system, while the voltage between the right and left edges of the system is kept constant. Since a 2D system is considered, the whole current between the edges can be regarded as the effective conductance of the system. If there is no bridging cluster, the conductance is obviously 0 and the Kirchhoff equations are not solved.

For a random 2D register network at the critical density, which is close to the critical density of the conventional bond-percolation model, $p=0.5$, it is reported ${ }^{14)}$ that the correlation length $\xi$ diverges as

$$
\xi \propto\left(p-p_{\mathrm{c}}\right)^{-v}
$$

where $v$ is a critical exponent, the value of which is independent of the details of the lattice. This divergence

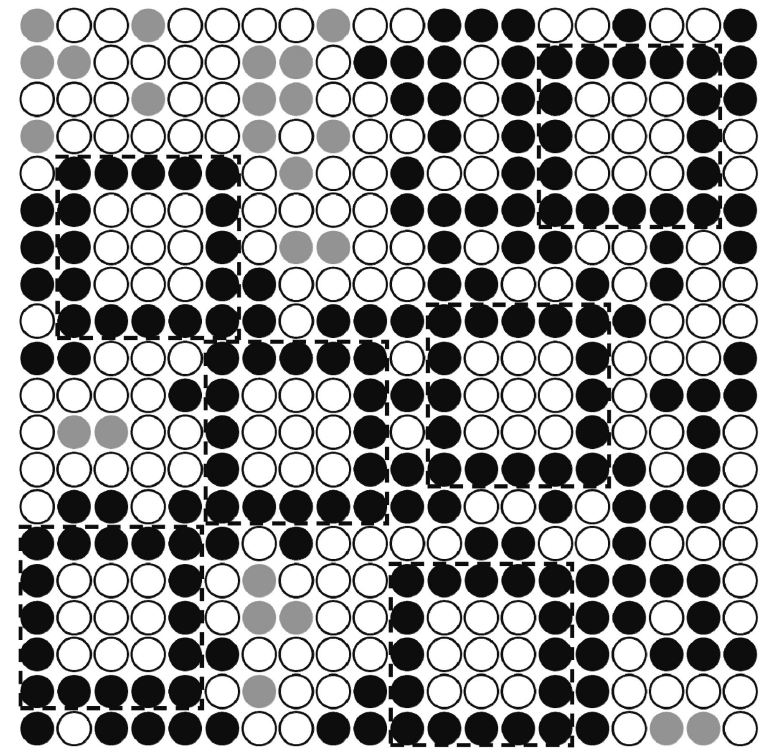

Fig. 2 This figure shows a typical particle configuration $(p=0.50$, $q=0.65$ ) with core-shell particles, whose surfaces are shown by the dashed lines. Note that this particular configuration is close to the percolation transition due to the effect of core-shell particles; the conducting particles unconnected to the largest cluster is only $12 \%$ (gray circles).

of $\xi$ (or disappearance of the "special" length scale) is widely utilized in the analysis of critical phenomena, for example in real-space renormalization methods. ${ }^{15-17)}$ Here, this is counter-intuitive because the diameter of the core-shell particle, $R$, is clearly a special length in the system we consider. However, if the system size, $L$, is large enough compared to $R$, the first few renormalization (or coarsegraining) operations reduce the core-shell configuration to ordinary conducting sites. Thus, we can analyze the coreshell condition in the same framework of the ordinary percolation.

The conductivity, denoted by $\sigma$, follows the relationship

$$
\sigma \propto\left(p-p_{\mathrm{c}}\right)^{\mu}
$$

where $\mu$ is an another exponent.

Considering the finite scaling hypothesis, such as

$$
\sigma(L, \xi)=\xi^{-\mu / \nu} S(L / \xi),
$$

where $\mathrm{S}()$ is an appropriate scaling function, we can derive that

$$
\sigma \propto L^{-\mu / v}
$$

for finite $L$, in the close vicinity of the critical point (diverging $\xi$ ). When the conductivity is plotted against the system size in a double-log manner, the criticality of the system is manifested by a linear plot and its slope has the value of $-\mu / \nu$.

The lattice sizes, $L$, used in the simulations are 100, 200, $300,400,500$ and 600 for calculation of critical exponent. Basically, the range of $p=0.580 \sim 0.628$ is scanned as the value of $p$, and the critical point is decided by the accordance of the result to eq. (5) (that is, the linearity of the plot). We used two designs of core-shell particles with the same shell thickness and different core size, as introduced in Fig 1. The 


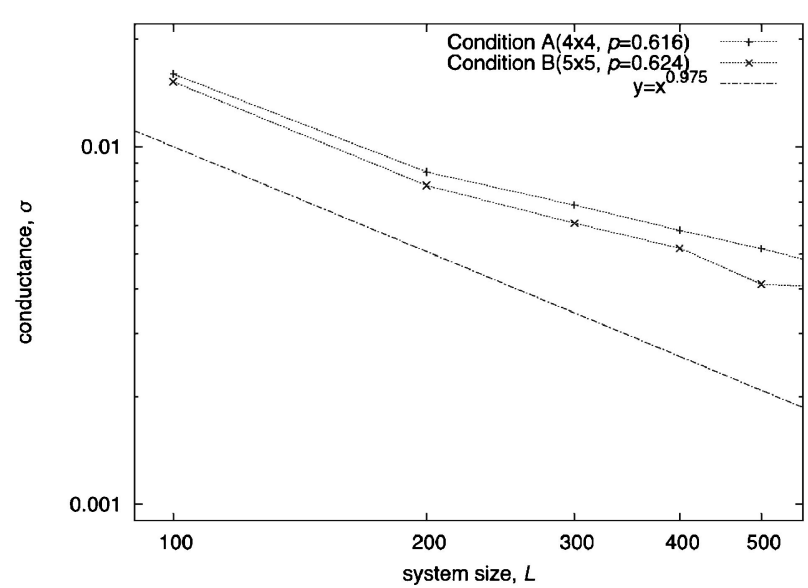

Fig. 3 Lattice size versus conductivity plots. The power-law dependence is clear, showing that finite-size scaling in the conductivity is not broken by the introduction of core-shell particles.

condition A used $4 \times 4$ core-shell particle and condition B uses $5 \times 5$ core-shell particles, along with the normal $(1 \times 1)$ particles. As mentioned above, the number of the core-shell particles are controlled in such a way that $50 \%$ of $q$ comes from core-shell particles. The data for conditions without insulating core is cited from out previous work (to be published elsewhere). We are also interested in the general level of the conductance because it is unlikely in real applications to employ granular materials very close to the critical density. For this, bigger lattice sizes, such as $1000 \times 1000$ and $2000 \times 2000$ are also used with much larger $p$. Typically, 1000 8000 randomly generated configurations are calculated, depending on the size of the lattice.

\section{Results and Discussion}

Our result in Fig. 3 shows that the effective conductance of our model exhibits roughly the same power-law behavior observed for the standard random network model, but with evidently different values of critical point. This difference can be attributed to the altered statistical nature of the network: unlike the case of conventional random register network, there is a clear short-range $(\sim R)$ correlation in the spatial distribution of conducting sites due to the core-shell particles.

The following is, to the author's best knowledge, the first report on the critical behavior of the "conductivity version" of the site percolation model involving the core-shell condition. The conductivity versus lattice size plot shows the best linearity at $p=0.616$ for condition A and $p=0.624$ for condition $\mathrm{B}$. These values are clearly different from the critical point of the conductivity version of bond-percolation, $p=0.500$, and slightly increased from that of the ordinary version of site-percolation $(p=0.593)$. The value of the critical exponent estimated by the slopes in Fig. 3 is roughly matched and slightly reduced than the value of critical exponents reported for "conductivity versions" of bondpercolation (0.975) for 2D. ${ }^{18)}$ So this model is not in a contradiction to the universality class of ordinary bondpercolation.

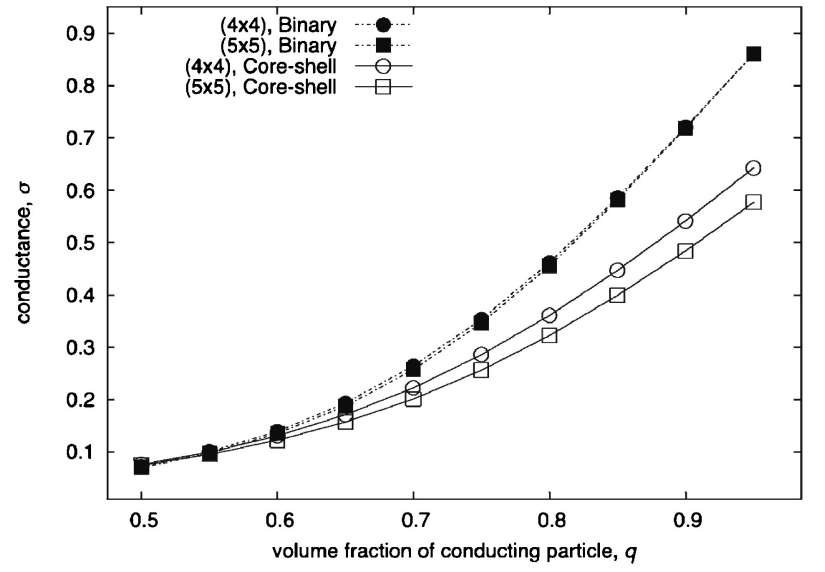

Fig. 4 The conductance as functions of the number fraction of sites occupied by conducting particles (that is, core, shell and individual conducting sites), $q$. The films with core-shell particles (open legends) exhibit greatly reduced conductance than the films without core-shell particles (filled legends). The larger the core is, the larger the reduction in the conductance becomes.

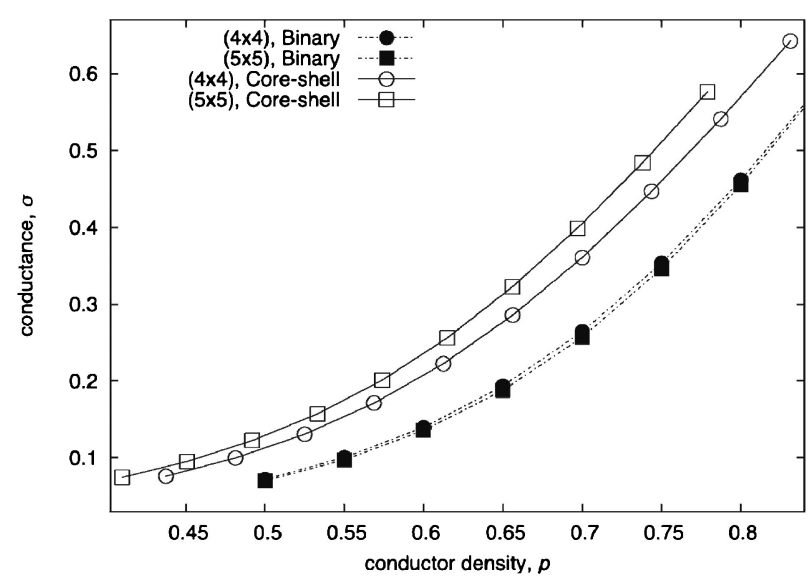

Fig. 5 The conductance as functions of the number fraction of conducting sites (that is, no core), $p$. The films with core-shell particles (open legends) exhibit greatly increased conductance for the same amount of conducting sites compared to the cases without core-shell particles (filled circles). The larger the core is, the larger the efficiency of the conduction.

The general (not close to the critical region) behavior of the effective conductance is shown in Figs. 4 and 5. The conducting particles with core-shell structure are expected to be a kind of "bridge" in the random register network and enhance the overall conductance, although there may be a strong negative effect imposed by the core on the "width" of the bridge, too.

According to Fig. 4, the percolating films with core-shell particles (open legends) have greatly reduced conductance compared to the normal cases with the same particle size composition and $q$ (filled legends). This is a direct consequence of the introduction of the insulator core, and the larger reduction of conductance is observed for larger core with the same shell thickness. However, as shown in Fig. 5, the percolating films with core-shell particles have largely increased conductance per conducting sites (that is, for the same $p$ ). And this effect of increasing conductance per unit amount of conducting material becomes larger when the size 
of the core is increased. It seems the introduction of coreshell particles into conducting film is effective for realizing much more efficient spatial configuration of conducting sites. Because core-shell particle impose a limit to the maximum possible density of the conducting site, the maximum conductance is limited in the films with core-shell particles (Fig. 5). In conclusion, the core-shell particles are effective way to improve conducting films in terms of saving conductor amount, unless maximum electric current required in the application is very large.

\section{Conclusion}

To aim for a better understanding of electric transport in thin film and an advanced design of conductive film with technologically crucial applications, a percolation model involving particles with core-shell design is, for the first time, investigated. The effects of core-shell particles are taken into account by regarding them as a special type of correlation in the quenched randomness in a random register network model. The conductance shows a powerlaw behavior just like the conventional random register models, however at increased critical points. The critical behavior of this new type of percolation phenomena is for the first time observed and roughly matched to the previous reports for similar model without core-shell particles.

Outside of the critical region, the conductivity per amount of conducting material $(\propto p)$ exhibits a strong increase suggesting that the introduction of core-shell particles into granular conductor-insulator mixture is a feasible way to improve the spatial distribution of the conductor particles in the composite materials without increasing the cost. Although other methods to realize similar effects on the spatial structure of granular materials are already reported, ${ }^{19)}$ it is noteworthy that employing core-shell design is one of the simplest methods to modulate such a microscopic structure of the target materials.

\section{Acknowledgement}

This work has been supported by Ministry of Economy, Trade and Industry (METI) under "Development for reduction of Indium in a transparent electrode ITO". The authors gratefully acknowledge the Center for Computational Materials Science at the IMR Tohoku University.

\section{REFERENCES}

1) R. B. H. Tahar, T. Ban, Y. Ohya and Y. Takahashi: J. Appl. Phys. 82 (1997) 865-870.

2) K. Nishio, S. Miyake, T. Sei, Y. Watanabe and T. Tsuchiya: J. Mater. Sci. 31 (1996) 3651-3656.

3) A. Tsunashima, H. Yoshimizu, K. Kodaira, S. Shimada and T. Matsushita: J. Mater. Sci. 21 (1986) 2731-2734.

4) T. D. Senguttuvan and L. K. Malhotra: Thin Solid Films 289 (1996) 22-28.

5) C. Terrier, J. P. Chatelon and J. A. Roger: Thin Solid Films 295 (1997) 95-100.

6) R. Sahara, H. Mizuseki, K. Ohno and Y. Kawazoe: Mater. Trans. JIM 40 (1999) 1314-1318

7) R. Sahara, H. Mizuseki, K. Ohno and Y. Kawazoe: J. Phys. Soc. Japan 68 (1999) 3755-3758.

8) M. K. Phani and D. Dhar: J. Phys. A 17 (1984) L645.

9) D. Dhar: Physica A 242 (1997) 341-346.

10) B. Agoudjil, L. Bos, J. C. Majeste, Y. Candau and Y. P. Mamunya: Composites Part a-Appl. Sci. Manufact. 39 (2008) 342-351.

11) H. B. Dai, H. X. Li and F. H. Wang: Surf. Coat. Technol. 201 (2006) 2859-2866.

12) I. Krupa, G. Mikova, I. Novak, I. Janigova, Z. Nogellova, F. Lednicky and J. Prokes: Euro. Polymer J. 43 (2007) 2401-2413.

13) S. Kirkpatrick: Rev. Modern Phys. 45 (1973) 574-588.

14) M. Sahimi, B. D. Hughes, L. E. Scriven and H. T. Davis: J. Phys. C 16 (1983) L521-L527.

15) A. B. Harris, T. C. Lubensky, W. K. Holcomb and C. Dasgupta: Phys. Rev. Lett. 35 (1975) 327-330.

16) C. Dasgupta: Phys. Rev. B 14 (1976) 1221-1224.

17) R. Riera, P. M. Oliveira, C. M. Chaves and S. L. A. D. Queiroz: Phys. Rev. B 22 (1980) 3481-3485.

18) J. M. Normand, H. J. Herrmann and M. Hajjar: J. Statist. Phys. 52 (1988) 441-446.

19) N. Johner, C. Grimaldi, T. Maeder and P. Ryser: Phys. Rev. E 79 (2009) 020104. 